\title{
La dinámica del empleo rural en el Brasil: un análisis mediante modelos de panel dinámico
}

\author{
Evânio Mascarenhas Paulo, Francisco José Silva Tabosa, \\ Ahmad Saeed Khan y Leonardo Andrade Rocha
}

\section{Resumen}

En este artículo se abordan algunos aspectos de la calidad del empleo en el Brasil, en particular el empleo rural. Se parte de la percepción de cambios en las relaciones de producción en el campo y sus repercusiones en las relaciones de trabajo. Se aplica una metodología de datos de panel que capta los condicionantes de esta calidad y se propone un índice de calidad del empleo. El universo rural constituye un ambiente más precario que el urbano, aunque las diferencias estén disminuyendo con el tiempo. Respecto del empleo agrícola, el crecimiento económico de la década de 2000 se traduce solo en la extensión de las condiciones existentes a los nuevos contratos, sin modificar la calidad media del empleo. El aumento de los ingresos del trabajo y el nivel de educación son instrumentos importantes para aumentar los niveles de calidad del empleo y superar problemas como la heterogeneidad entre grupos.

\section{Palabras clave}

Empleo rural, mercado de trabajo, zonas rurales, relaciones laborales, condiciones de trabajo, medición, evaluación, estadísticas de empleo, modelos econométricos, Brasil

\section{Clasificación JEL}

C23, J43, R11

\section{Autores}

Evânio Mascarenhas Paulo es Profesor del Departamento de Economía de la Universidad Regional del Cariri (Brasil). Correo electrónico: evanio.paulo@urca.br.

Francisco José Silva Tabosa es Profesor del Programa de Posgrado en Economía Rural del Departamento de Economía Agrícola de la Universidad Federal de Ceará (Brasil). Correo electrónico: franzetabosa@ufc.br.

Ahmad Saeed Khan es Profesor del Programa de Posgrado en Economía Rural del Departamento de Economía Agrícola de la Universidad Federal de Ceará (Brasil). Correo electrónico: saeed@ufc.br.

Leonardo Andrade Rocha es Profesor del Departamento de Ciencias Humanas de la Universidad Federal Rural del Semi-Árido (Brasil). Correo electrónico: leonardoandrocha@yahoo.com.br. 


\section{Introducción}

Los procesos que han tenido lugar en el mundo rural y agrícola en los últimos años son de tal complejidad y magnitud que se han propagado por diversas estructuras propias de estos espacios. Además de fenómenos específicos del mundo rural, como la mecanización y la modernización de la agricultura, algunos cambios vinculados al sector urbano, como el estado de semiletargo de las actividades industriales, incidieron fuertemente en el sector rural (Da Silva, 1997).

En este contexto, se puede esperar que se produzca un cambio drástico y dramático en las estructuras que componen los sistemas agrícola y rural del Brasil. En este artículo se aborda una de ellas en particular: el mercado de trabajo. Es de esperar que el mercado de trabajo refleje los cambios registrados en la agricultura y el medio rural en los últimos años como una especie de "espejo" de los nuevos sistemas y técnicas de producción (Carneiro, 1998).

Naturalmente, los nuevos procesos productivos que comienzan a aplicarse en la agricultura (que están más alineados con las modernas técnicas de acumulación capitalista) cambiarán las formas de contratación y el número de trabajadores necesarios para mover los medios de producción en la agricultura (Campolina, Silveira y De Magalhães, 2009). En la literatura, el aumento de la reserva de mano de obra que se libera en virtud de los cambios en los procesos agrícolas y no es absorbida por las actividades industriales y de servicios en el medio urbano se plantea como una consecuencia inmediata de estos nuevos fenómenos en el mercado de trabajo rural. Así, se considera que el movimiento espacial de trabajadores del campo a la ciudad es una consecuencia esperada de estos procesos. Sin embargo, en el último período no se asiste al fin del éxodo rural que caracterizaba las décadas anteriores, sino a su reformulación, en la medida en que se observa una significativa reducción de las migraciones directas del campo a la ciudad y un aumento igualmente significativo de las "migraciones sectoriales" del trabajo agrícola al trabajo no agrícola, con un conjunto de elementos que estimulan estos movimientos (Balsadi, 2007).

La mitigación de los factores que incentivan a la población rural a migrar a las ciudades representa un punto de inflexión importante en la relación entre las zonas rurales y urbanas. Sin embargo, las diferencias de ingresos y condiciones generales de trabajo entre el sector primario tradicional y los demás sectores de la economía estimulan la migración entre sectores. Así, el impacto y la influencia de las actividades no agrícolas en el mercado de trabajo rural pasaron a ser cada vez mayores. En la actualidad los trabajadores no necesitan desvincularse del medio espacial de sus hogares en las zonas rurales, sino que están cada vez más vinculados a actividades no agrícolas que no son inherentes a la agricultura pero están asociadas a esta (Balsadi y Da Silva, 2008).

Por lo expuesto, en este artículo se procura analizar, en términos generales, los elementos del patrón de calidad del empleo de los trabajadores asalariados rurales y urbanos en el Brasil, considerando las nuevas determinaciones del espacio rural y sus consecuencias en las relaciones de trabajo en el campo. En particular, se examinan los cambios recientes en el mercado de trabajo rural, con énfasis en los efectos provocados por las transformaciones en los espacios rurales, así como verificar el nivel de calidad de las ocupaciones rurales y urbanas en el Brasil sobre la base del índice de calidad del empleo. Por último, se busca determinar los factores condicionantes de la calidad de estas ocupaciones para los trabajadores asalariados en el Brasil mediante el modelo de datos de panel.

Dadas las necesidades del estudio, que requiere una base de datos detallada y con continuidad en el tiempo, se utiliza esencialmente información de naturaleza secundaria procedente de bases de datos de institutos nacionales de investigación, en particular del Instituto Brasileño de Geografía y Estadística (IBGE).

El estudio se divide en cinco secciones, incluida esta introducción. En la segunda sección se definen los métodos y las estrategias de estudio y se discuten los aspectos conceptuales y metodológicos. En 
la tercera sección se presentan y examinan los resultados descriptivos del índice de calidad del empleo y en la cuarta se analizan los resultados empíricos con la presentación de los modelos econométricos propuestos. Por último, se presentan los comentarios finales de la investigación.

\section{Aspectos conceptuales y metodológicos}

\section{Base de datos y construcción del índice de calidad del empleo}

Los datos utilizados en el estudio se tomaron de la encuesta nacional de hogares (PNAD) y corresponden al período comprendido entre 2004 y 2014. En este período se consolidan algunos cambios en el mundo del trabajo y por eso es importante para el análisis del mercado laboral brasileño reciente, en particular del empleo rural. La década de 2000 constituye el escenario de fenómenos como el crecimiento de la masa de trabajadores y del ingreso medio del trabajo. Estas transformaciones son el resultado de una especie de "metamorfosis" de las formas de contratación y reclutamiento y de una mayor demanda de mano de obra más calificada y preparada que induce un proceso de competencia, tanto entre los empleadores como entre los empleados, con sus respectivos efectos en la calidad de las ocupaciones.

El objetivo del trabajo consiste en determinar los factores que condicionan la calidad de las ocupaciones para cuatro grandes grupos de población específicos. El primero corresponde a los empleados asalariados agrícolas urbanos, es decir las personas empleadas en condición de asalariadas (tanto formales como informales) en empresas del grupo agrícola y residentes en áreas urbanas. El segundo grupo está formado por los trabajadores no agrícolas urbanos, que son los asalariados de empresas no agrícolas residentes en regiones urbanas. El tercer grupo está integrado por los trabajadores asalariados dedicados a actividades agrícolas que viven en áreas rurales y, por último, el cuarto grupo corresponde a los empleados rurales de empresas no agrícolas.

El corte elegido se refiere solo al empleo asalariado, pues el análisis de su naturaleza y características (formalidad, horas de trabajo, seguridad, entre otras) es más asequible que el de otros tipos de empleo. Si bien esto permite una mejor caracterización de este segmento del mercado laboral, los diversos segmentos del mercado de trabajo tienen características singulares y, por tanto, dinámicas de calidad del empleo también diferentes. Además, los distintos patrones de segmentación de la calidad ocupacional se afectan mutuamente, pues la calidad del empleo asalariado influye en los otros segmentos del mercado de trabajo y resulta influenciada por estos.

En parte, la calidad ocupacional refleja el ambiente y las oportunidades de empleo que se presentan de modo distinto en cada categoría de segmentación, lo que produce patrones de calidad muy heterogéneos para los diferentes segmentos. Así, un cambio en la composición del mercado de trabajo, como por ejemplo una mayor o menor participación del trabajo asalariado, tiene efectos en la calidad ocupacional en su conjunto. Asimismo, esto puede inducir procesos que influyen, a su vez, en la calidad de los estratos del mercado laboral, incluido el propio trabajo asalariado. Cabe señalar que la terminología de calidad ocupacional desarrollada en este trabajo no capta estas interacciones de la dinámica de segmentación del mercado de trabajo.

Para Do Nascimento y otros (2008), el concepto de calidad del empleo puede variar según diversos aspectos. Sin embargo, lo que se pretende en esta investigación es, a partir de variables sobre el mercado de trabajo, medir los efectos de algunos elementos que, a juicio de los autores, son relevantes en la determinación de la calidad del empleo. Estos incluyen la inexistencia de trabajo infantil, la regularidad de las horas de trabajo semanales sin presencia de sobrecarga laboral, el contrato de trabajo, la contribución a institutos de seguridad social públicos o privados y el nivel de ingresos, además 
de aspectos relativos al nivel de educación de los trabajadores. No obstante, la calidad ocupacional supone factores difíciles de abordar, relacionados con aspectos como la insalubridad, la exposición al riesgo y la inspección de las condiciones de trabajo por la justicia laboral, entre otros. Esto también se debe tener en cuenta cuando se trabaja con datos regionalizados.

El índice de calidad del empleo propuesto en este trabajo es una adaptación del índice formulado inicialmente por Balsadi (2007), que constituye la matriz inicial. El índice de calidad del empleo se basa en un sistema de puntuación, en el que a cada franja de la subcategoría del índice se atribuye un valor de 0 a 4 . En esta escala, 0 representa la peor condición y 4 la mejor. El método de construcción de los índices se detalla en el cuadro 1.

Cuadro 1

Metodología de construcción del índice de calidad del empleo

\begin{tabular}{|c|c|c|c|c|c|}
\hline Tasa de escolaridad & $\begin{array}{l}\text { [0] Sin instrucción } \\
\text { o con educación } \\
\text { primaria, con salario } \\
\text { de subsistencia }\end{array}$ & $\begin{array}{l}\text { [1] Caso anterior } \\
\text { sin restricción }\end{array}$ & $\begin{array}{l}\text { [2] Educación } \\
\text { primaria a educación } \\
\text { secundaria }\end{array}$ & $\begin{array}{l}\text { [3] Educación superior } \\
\text { y de posgrado }\end{array}$ & $\begin{array}{l}\text { [4] Caso anterior } \\
\text { con salario alto }\end{array}$ \\
\hline $\begin{array}{l}\text { Horas de trabajo } \\
\text { por semana } \\
{[0,15]}\end{array}$ & [0] Más de 44 horas & $\begin{array}{l}\text { [1] Entre } 40 \\
\text { y } 44 \text { horas }\end{array}$ & $\begin{array}{l}\text { [2] Entre } 30 \\
\text { y } 39 \text { horas }\end{array}$ & [3] Entre 1 y 29 horas & $\begin{array}{l}\text { [4] Caso anterior } \\
\text { con salario alto }\end{array}$ \\
\hline $\begin{array}{l}\text { Edad del trabajador } \\
{[0,23]}\end{array}$ & $\begin{array}{c}\text { [0] De } 0 \text { a } 15 \\
\text { años de edad } \\
\text { (trabajo infantil) }\end{array}$ & $\begin{array}{l}\text { [1] De } 16 \text { a } 18 \\
\text { años de edad }\end{array}$ & $\begin{array}{l}\text { [2] De } 19 \text { a } 24 \text { años } \\
\text { de edad, con bajo } \\
\text { nivel de educación } \\
\text { e informal }\end{array}$ & $\begin{array}{l}\text { [3] Caso anterior } \\
\text { sin restricción }\end{array}$ & $\begin{array}{l}\text { [4] Más de } 25 \\
\text { años de edad }\end{array}$ \\
\hline $\begin{array}{l}\text { Remuneración } \\
{[0,30]}\end{array}$ & $\begin{array}{l}\text { [0] Salario inferior a } \\
\text { un salario mínimo } \\
\text { (subsistencia) }\end{array}$ & $\begin{array}{l}\text { [1] De uno a dos } \\
\text { salarios mínimos }\end{array}$ & $\begin{array}{l}\text { [2] De dos a tres } \\
\text { salarios mínimos }\end{array}$ & $\begin{array}{l}\text { [3] De tres a cinco } \\
\text { salarios mínimos }\end{array}$ & $\begin{array}{l}\text { [4] Más de cinco } \\
\text { salarios mínimos }\end{array}$ \\
\hline $\begin{array}{l}\text { Trabajo informal } \\
{[0,12]}\end{array}$ & $\begin{array}{l}\text { [0] Sin contribución, } \\
\text { sin contrato de trabajo } \\
\text { y con bajos ingresos }\end{array}$ & $\begin{array}{l}\text { [1] Caso anterior } \\
\text { sin restricción }\end{array}$ & $\begin{array}{l}\text { [2] Sin contribución y } \\
\text { con contrato de trabajo }\end{array}$ & $\begin{array}{l}\text { [3] Con contribución y } \\
\text { sin contrato de trabajo }\end{array}$ & $\begin{array}{l}\text { [4] Con contribución } \\
\text { y contrato de trabajo }\end{array}$ \\
\hline
\end{tabular}

Fuente: Elaboración propia, sobre la base de O. V. Balsadi, "Qualidade do emprego na agricultura brasileira no período 2001-2004 e suas diferenciações por culturas", Revista de Economia e Sociologia Rural, vol. 45, № 2, abril-junio de 2007.

Los subíndices seleccionados fueron los siguientes: i) nivel de educación de los trabajadores, intercalado con bajos ingresos, a fin de verificar la relación entre la baja calidad ocupacional y el menor grado de instrucción de los trabajadores; ii) horas de trabajo, que mide los diferentes niveles de horas trabajadas y permite una percepción de la sobrecarga de trabajo; iii) edad del trabajador, con miras a detectar la presencia de trabajo infantil y los diferentes niveles de vulnerabilidad a que están expuestos los trabajadores jóvenes; iv) remuneración media, considerando que la tasa media de remuneración condiciona la percepción de la calidad ocupacional a la que los trabajadores están expuestos; y v) tipo de vínculo, que revela la presencia de trabajo temporal y ocasional, intercalado con bajos niveles de remuneración. El índice de calidad del empleo es esencialmente una media ponderada de las categorías descritas, que captan aspectos relevantes para la percepción de la calidad del puesto de trabajo.

De acuerdo con Do Nascimento y otros (2008), el índice de calidad del empleo se obtiene a partir de la media ponderada de los indicadores parciales. El peso de cada categoría se detalla en la primera columna del cuadro 1. También según los autores, el peso de cada categoría para la composición del índice de calidad del empleo refleja las contribuciones relativas y se estableció mediante el sistema convencional de pesos, es decir, por el propio proponente del índice, a partir de un sistema de prioridades. Por lo tanto, la ponderación puede generar controversias, porque siempre implica un cierto grado de arbitrariedad de los autores, dada la importancia atribuida a cada indicador parcial. Sobre la base de estos indicadores, el índice de calidad del empleo se construye a partir de las medias ponderadas de los indicadores parciales. De este modo, teniendo en cuenta las debidas arbitrariedades, estos indicadores pueden contribuir al análisis de la calidad del empleo rural y urbano, que permite asimilar con mayor detalle las condiciones del trabajo asalariado y establecer comparaciones. 


\section{Descripción de las variables}

Para determinar la calidad de las ocupaciones de los grupos seleccionados en esta investigación, se construyó un conglomerado de variables que procuran sintetizar los factores condicionantes del patrón de calidad de las relaciones de trabajo. Estas variables se resumen en el cuadro 2.

Cuadro 2

Grupo de variables, descripción y fuentes

\begin{tabular}{|c|c|c|}
\hline Variables & $\begin{array}{c}\text { Código } \\
\text { de la variable }\end{array}$ & Fuente \\
\hline Logaritmo natural del índice de calidad del empleo & QE & Elaboración propia \\
\hline Logaritmo natural del índice de calidad del empleo rezagado & $\mathrm{QE}_{t-1}$ & Variable endógena \\
\hline Logaritmo natural del valor agregado agrícola & VA01 & $\begin{array}{c}\text { Instituto Brasileño de Geografía } \\
\text { y Estadística (IBGE) }\end{array}$ \\
\hline Logaritmo natural del valor agregado no agrícola & VA02 & IBGE \\
\hline $\begin{array}{l}\text { Logaritmo natural del ingreso medio del trabajo para cada } \\
\text { unidad de la federación (trabajadores agrícolas urbanos) }\end{array}$ & RM01 & IBGE \\
\hline $\begin{array}{l}\text { Logaritmo natural del ingreso medio del trabajo para cada unidad } \\
\text { de la federación (trabajadores no agrícolas urbanos) }\end{array}$ & RM02 & IBGE \\
\hline $\begin{array}{l}\text { Logaritmo natural del ingreso medio del trabajo para cada unidad } \\
\text { de la federación (trabajadores no agrícolas rurales) }\end{array}$ & RM03 & IBGE \\
\hline $\begin{array}{l}\text { Logaritmo natural del ingreso medio del trabajo para cada } \\
\text { unidad de la federación (trabajadores agrícolas rurales) }\end{array}$ & RM04 & IBGE \\
\hline Logaritmo natural del índice de concentración de Gini para el ingreso del trabajo & IG & Elaboración propia \\
\hline $\begin{array}{l}\text { Logaritmo natural de la participación de las personas que han completado } \\
\text { al menos la educación secundaria en cada unidad de la federación }\end{array}$ & EDU & IBGE \\
\hline
\end{tabular}

Fuente: Elaboración propia.

La primera variable descrita (QE) se refiere al índice de calidad del empleo, cuya construcción se explicó en la sección anterior. Como se vio, el índice recoge información sobre el nivel de formalización de los contratos de trabajo y el patrón de ingresos de los trabajadores. De esta manera, proporciona un indicio del grado de complejidad de las relaciones de trabajo. Con esta investigación se busca verificar las posibles diferencias entre las condiciones de ocupación de la mano de obra en el campo y en la agricultura, en comparación con las relaciones contractuales en las áreas urbanas y en las actividades de los segmentos no agrícolas. Así, se busca determinar sus condicionantes y sus contribuciones a la calidad del empleo.

La siguiente variable descrita $\left(Q E_{t-1}\right)$ es el índice de calidad del empleo rezagado, que es una característica del tipo de modelización econométrica elegido en esta investigación para evaluar las propiedades de las relaciones de trabajo entre los grupos estudiados. Se espera que esta variable sea estadísticamente significativa en la medida en que la calidad del empleo en el período anterior resulte determinante de la calidad del empleo futuro. Esta esperanza se debe al movimiento natural de la dinámica del mercado de trabajo, en el que las relaciones laborales suelen volverse más complejas y avanzadas a lo largo del tiempo, de manera que - en un ambiente de crecimiento económico como el observado durante los años de referencia - las posibilidades de retroceder a un patrón inferior son remotas. Asimismo, vistas la presencia y las especificidades de la legislación laboral (que fija un estándar en materia de relaciones contractuales que comienza a aplicarse en el mercado), el retroceso de esos derechos podría suponer el incumplimiento de las leyes laborales.

La variable VA01 representa el valor agregado de las actividades agropecuarias de cada estado. Uno de los posibles comportamientos de esta variable es que el aumento del nivel de bienes producidos por el conjunto de las actividades agrícolas de los estados sea positivo y tienda a aumentar la calidad del empleo. La lógica consiste en que el crecimiento de la agricultura y la ganadería determinaría un incremento de la demanda de mano de obra, aumentando el nivel de ingresos de los trabajadores 
y mejorando las condiciones de trabajo. Otro comportamiento que cabe esperar es que el nivel de actividad no esté relacionado con el aumento de la calidad del mercado de trabajo. Así, el crecimiento económico solo supondría la extensión a los nuevos contratados de las relaciones contractuales ya existentes, sin modificaciones en su estructura. La evaluación de los signos y los niveles de significación de los coeficientes de esta variable permitirá determinar cuál de esas hipótesis es la más conveniente.

En el caso de la variable VA02, que expresa el valor agregado de las actividades no agrícolas, se espera que tenga un efecto positivo (negativo) en la calidad de las ocupaciones, en la medida en que el incremento de los puestos de trabajo - que en el caso de las actividades no agrícolas suelen ser más complejos y de mayor calidad que las ocupaciones puramente agrícolas - induzca un aumento (reducción) de la calidad del empleo. Además, como en el caso anterior, la variable en cuestión puede no estar relacionada con el aumento de la calidad de los contratos de trabajo por no suponer cambios en las relaciones de contratación. Aquí también se busca evaluar el comportamiento de los efectos cruzados, es decir, el efecto del crecimiento económico de las actividades no agrícolas en la dinámica del empleo agrícola y rural y el efecto del crecimiento de las actividades agrícolas en la calidad del empleo no agrícola y urbano.

El grupo de variables "RM" expresa el nivel de ingreso medio del trabajo principal para cada grupo en cada unidad de la federación. Así, RM01 expresa el nivel de ingreso medio de los trabajadores agrícolas urbanos; RM02 el ingreso medio de los trabajadores no agrícolas urbanos; RM03 el ingreso medio de los trabajadores no agrícolas rurales y, por último, RM04 el ingreso medio de los trabajadores agrícolas rurales.

La variable "IG" corresponde al índice de Gini, que mide el nivel de concentración del ingreso en cada unidad de la federación. Esta variable evalúa la manera en que se distribuye la estructura del ingreso. El signo negativo esperado se justifica por la percepción de que a medida que aumenta el patrón de concentración del ingreso, tiende a disminuir la calidad de las ocupaciones. Se supone además que, si este coeficiente es insignificante, la calidad del trabajo es independiente de la estructura de concentración del ingreso en el mercado de trabajo. Así, el crecimiento del ingreso garantiza, por sí solo, el crecimiento de la calidad de las ocupaciones, aunque esto no signifique cambios en la estructura de concentración.

Por último, la variable "EDU" expresa el nivel de participación de las personas que han completado al menos la educación secundaria en cada unidad de la federación. Se espera que cuanto más alto sea el nivel de educación, mayor sea la eficiencia del trabajo y que, a medida que crezca el nivel general de educación, aumente también el patrón de calidad del trabajo.

\section{a) Método generalizado de momentos (MGM)}

Para evaluar el comportamiento de la calidad del empleo agrícola y no agrícola - tanto urbano como rural - y sus determinantes en un sistema de datos de panel dinámico, pues se necesita la presencia de la variable dependiente rezagada como variable explicativa (Baltagi, 2009), se emplean los estimadores del método generalizado de momentos para sistemas (MGM-sistema) desarrollado en los trabajos de Arellano y Bond (1991), Arellano y Bover (1995) y Blundell y Bond (1998).

En resumen, en esta investigación se procura estimar modelos compuestos por las cuatro ecuaciones especificadas en los siguientes modelos de regresión para datos de panel:

$$
\begin{aligned}
& \ln \left(Q E_{A U}\right)=\beta_{1} \ln \left(Q E_{t-1}\right)+\beta_{2} \ln (A V 01)+\beta_{3} \ln (A V 02)+\beta_{4} \ln (R M 01)+\beta_{5} \ln (I G)+\beta_{6} \ln (E D U)+V_{t}+\mu_{i t} \\
& \ln \left(Q E_{N U}\right)=\beta_{1} \ln \left(Q E_{t-1}\right)+\beta_{2} \ln (A V 01)+\beta_{3} \ln (A V 02)+\beta_{4} \ln (R M 02)+\beta_{5} \ln (I G)+\beta_{6} \ln (E D U)+V_{t}+\mu_{i t}
\end{aligned}
$$




$$
\begin{aligned}
& \ln \left(Q E_{N R}\right)=\beta_{1} \ln \left(Q E_{t-1}\right)+\beta_{2} \ln (A V 01)+\beta_{3} \ln (A V 02)+\beta_{4} \ln (R M 03)+\beta_{5} \ln (I G)+\beta_{6} \ln (E D U)+V_{t}+\mu_{i t} \\
& \ln \left(Q E_{A R}\right)=\beta_{1} \ln \left(Q E_{t-1}\right)+\beta_{2} \ln (A V 01)+\beta_{3} \ln (A V 02)+\beta_{4} \ln (R M 04)+\beta_{5} \ln (I G)+\beta_{6} \ln (E D U)+V_{t}+\mu_{i t}
\end{aligned}
$$

En que la variable dependiente es el índice de calidad del empleo (QE) de cada unidad de la federación y sus subíndices se refieren al empleo agrícola urbano (AU), el empleo no agrícola urbano (NU), el empleo no agrícola rural (NR) y el empleo agrícola rural (AR) respectivamente; $Q E_{t-1}$ expresa el índice de calidad del empleo rezagado en un año, y en cada caso esa variable representa la variable dependiente rezagada para cada grupo (la introducción de esta variable es una característica de este tipo de modelización econométrica); "VA01" representa el valor agregado en las actividades agrícolas de cada unidad de la federación; "VA02" expresa el valor agregado en las actividades no agrícolas; "RM01" representa el ingreso medio en cada estado en las actividades agrícolas urbanas en la primera ecuación; "RM02" representa el ingreso medio en las actividades no agrícolas urbanas en la segunda ecuación; "RM03" representa el ingreso medio en las actividades no agrícolas rurales en la tercera ecuación; "RM04" representa el ingreso medio en las actividades agrícolas rurales en la cuarta ecuación, "IG" expresa el nivel de concentración del ingreso para cada estado medido por el índice de Gini; "EDU” representa la participación de las personas que han completado al menos la educación secundaria en cada unidad subnacional; $V_{i}$ los efectos no observables de los individuos y, $\mu_{i t}$ representa las perturbaciones aleatorias. Los subíndices $i$ y $t$ se refieren, respectivamente, al i-ésimo estado en el año $t$.

Estos modelos tienen las siguientes hipótesis: $E\left[v_{i}\right]=E\left[\mu_{i t}\right]=E\left[v_{i t} \mu_{i t}\right]=0$ para $i=1,2, \ldots, n$; $t=1,2, \ldots, t$. Además, se supone que el error no está correlacionado temporalmente, es decir $E / \mu_{i, t}, \mu_{i, s} /=0$ para $i=1,2, \ldots, n \forall t \neq s$. También existe la hipótesis estándar relativa a las condiciones iniciales $E\left|Q E_{t-1} \mu_{i t}\right|=0$ para $i=1,2, \ldots, n$ y $t=1,2, \ldots, t$. Estas hipótesis son válidas para todas las ecuaciones de los modelos presentados anteriormente.

En los trabajos presentados en la literatura, especialmente el de Arellano y Bond (1991), se destacan algunos problemas a la hora de estimar los modelos especificados anteriormente mediante las técnicas de estimación tradicionales, debidos a:

- la presencia de los efectos no observables de los individuos, $V_{t}$, junto con la variable dependiente rezagada en un período, $Q E_{t-1}$, en el lado derecho de la ecuación. En este caso, la omisión de los efectos fijos individuales en el modelo de panel dinámico hace que los estimadores de mínimos cuadrados ordinarios (MCO) sean sesgados e inconsistentes. Sin embargo, el estimador dentro de los grupos (within groups), que corrige la presencia de efectos fijos, genera una estimación de $\beta_{1}$ sesgada a la baja en paneles con una dimensión temporal pequeña; y

- la probable endogeneidad de las variables explicativas. En este caso, es necesario tratar la endogeneidad en el lado derecho de las ecuaciones para evitar un posible sesgo generado por el problema de simultaneidad.

Marinho y Araujo (2010) afirman que un posible método para intentar superar este problema sería eliminar la presencia de efectos fijos del modelo. Así, un primer intento consistiría en estimar los modelos mediante mínimos cuadrados ordinarios con variables ficticias para cada estado o mediante el método dentro de los grupos (within groups), que genera las mismas estimaciones que el método anterior pero con desviaciones estándar de los coeficientes ligeramente inferiores. Los estimadores del coeficiente por ambos métodos serán menores que los obtenidos por mínimos cuadrados ordinarios. Sin embargo, aún se podía mostrar que el sesgo en el panel dinámico seguía existiendo.

También según los mismos autores, otra manera de eliminar estos problemas sería tomar la primera diferencia de las ecuaciones anteriores y estimarlas con el método generalizado de 
momentos (MGM). Este método suele denominarse método generalizado de momentos en diferencias (MGM-diferenciado) y consiste en la eliminación de los efectos fijos mediante la primera diferencia de las ecuaciones anteriores. Así, la ecuación (1), por ejemplo, se transformó en la ecuación (5):

$$
\Delta \ln \left(Q E_{A U}\right)=\beta_{0}+\beta_{1} \Delta\left(\ln Q E_{t-1}\right)+\beta_{2} \Delta(\ln V A 01)+\beta_{3} \Delta(\ln V A 02)+\beta_{4} \Delta(R M 01)+\beta_{5} \Delta(I G)+\beta_{6} \Delta(E D U)+V_{t}+\mu_{i t}
$$

De manera que, para cualquier variable, $Y_{i t}, \Delta \ln Y_{i t}=\ln Y_{i t}-\ln Y_{i t-1}$. Obsérvese que en las ecuaciones anteriores, $\triangle \ln Q E_{t-1}$ está correlacionada con los términos de error, $\Delta \ln \mu_{i t}$. En consecuencia, los estimadores de mínimos cuadrados ordinarios (MCO) para sus coeficientes serán sesgados e inconsistentes. Por lo tanto, es necesario utilizar variables instrumentales para $\triangle \ln Q E_{t-1}$ y en cada modelo.

Las hipótesis adoptadas en las ecuaciones presentadas al comienzo de esta sección implican que las condiciones de momentos $E\left[\Delta \ln Q E_{t-1} \Delta \ln \mu_{i t}\right]=0$ para $t=3,4, \ldots, n$ y $s \geq 2$ son válidas. Sobre la base de estos momentos, se debe emplear $\ln Q E_{t-1}$ para $t=3,4, \ldots, n$ y $s \geq 2$ como instrumentos para la ecuación en primera diferencia.

En el segundo caso, los valores de la variable rezagada en uno o más períodos son instrumentos válidos en la estimación de la ecuación y en el último caso los valores rezagados en dos o más períodos son instrumentos válidos en la estimación de esta ecuación.

De acuerdo con Arellano y Bover (1995) y Blundell y Bond (1998), estos instrumentos son débiles cuando las variables dependientes y explicativas presentan una fuerte persistencia y cuando la varianza relativa de los efectos fijos aumenta. Esto produce un estimador MGM-diferenciado sesgado e inconsistente para paneles con $t$ pequeño.

En consecuencia, Arellano y Bover (1995) y Blundell y Bond (1998) proponen un sistema que combina el conjunto de ecuaciones en diferencia - ecuaciones (1) a (4) - con el conjunto de ecuaciones en nivel -ecuación (5) - para reducir este problema de sesgo. Este sistema se denomina método generalizado de momentos para sistemas (MGM-sistema). Para las ecuaciones en diferencias, el conjunto de instrumentos es el mismo descrito anteriormente. Para la regresión en nivel, los instrumentos apropiados son las diferencias rezagadas de las respectivas variables. Por ejemplo, suponiendo que las diferencias de las variables explicativas no están correlacionadas con los efectos fijos individuales (para $t=3,4, \ldots, n$ ) $E\left[\Delta \ln Q E_{t-1} \mu_{i t}\right]=0$ para $i=1,2, \ldots, n$. Entonces, si las variables explicativas en diferencias y $\triangle \ln Q E_{t-1}$ son exógenas o débilmente exógenas, constituyen instrumentos válidos para la ecuación en nivel. Lo mismo ocurre si son endógenas, pero los instrumentos son las variables explicativas en diferencias rezagadas de un período y $\triangle \ln Q E_{t-1}$ considerado en este trabajo, conforme Marinho y Araujo (2010).

Las estimaciones del MGM-sistema son el resultado de la estimación con un estimador corregido para evitar que el respectivo estimador de las varianzas subestime las verdaderas varianzas en una muestra finita. El estimador utilizado se propuso en dos pasos. En la primera etapa, se supone que los términos de error son independientes y homocedásticos en los estados y a lo largo del tiempo. En la segunda etapa, los residuos obtenidos en la primera etapa se utilizan para construir una estimación consistente de la matriz de varianza-covarianza, relajando así las hipótesis de independencia y homocedasticidad. El estimador de la segunda etapa es asintóticamente más eficiente en relación con el estimador de la primera etapa (Marinho y Araujo, 2010).

Por último, existen dos tipos de pruebas para probar la robustez y la consistencia de los modelos. La prueba de Sargan se utilizó con el objetivo de verificar la validez de los instrumentos. Si no se rechaza la hipótesis nula, los instrumentos son robustos. Además, como se supone inicialmente que el error ite no está autocorrelacionado, se realiza una prueba de correlación serial de primer orden y otra de segundo orden sobre los residuos en primera diferencia, $\Delta \varepsilon_{i t}$. Se espera que estos errores estén correlacionados en primer orden y no autocorrelacionados en segundo orden (Marinho y Araujo, 2010). 


\section{Algunos resultados descriptivos}

Los datos que se presentan a continuación ilustran el caso del mercado de trabajo brasileño en los últimos años, en el que se observa la expansión de las ocupaciones no agrícolas entre la población urbana y rural del país. Esto refleja una importante transformación del patrón de organización del espacio rural en el Brasil, potenciada por los procesos de formación y crecimiento de las agroindustrias y su extrapolación a las áreas rurales y por el desarrollo de una nueva dinámica del sector de servicios, pues aunque una parte considerable de la población sigue viviendo en las zonas rurales, su ocupación principal no está directamente relacionada con la agricultura. Otro dato importante que surge del cuadro 3 es que el empleo rural total aumenta a medida que el empleo agrícola (urbano y rural) disminuye, lo que demuestra la migración sectorial de los trabajadores a las actividades no agrícolas.

Cuadro 3

Brasil: población empleada, por zona de residencia y tipo de actividad, 2004 y 2014

(En número de personas)

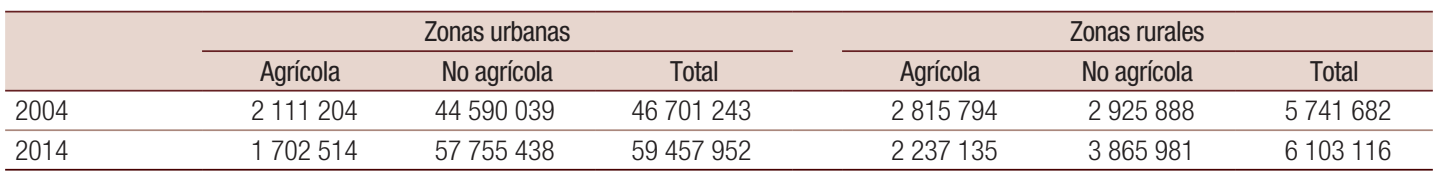

Fuente: Elaboración propia, sobre la base de Instituto Brasileño de Geografía y Estadística (IBGE), "Pesquisa Nacional por Amostra de Domicílios - PNAD" [en línea] https://www.ibge.gov.br/estatisticas/sociais/educacao/9127-pesquisanacional-por-amostra-de-domicilios.html?=\&t=o-que-e.

A continuación se presentan los índices de calidad de las ocupaciones para las unidades subnacionales del Brasil. En el mapa 1, que muestra el índice de calidad del empleo agrícola rural y urbano, se observa que los mejores niveles de calidad se registran en los estados del centro-sur del país, en particular en la región Centro-Oeste (especialmente Mato Grosso, Mato Grosso do Sul y Goiás). Junto con São Paulo y Paraná, esta presenta los mejores patrones de calidad del empleo agrícola, ya sea urbano o rural. Autores como Priori y otros (2012) añaden que, hasta hace poco tiempo, muchas economías regionales del Brasil estaban más insertadas en la lógica del mercado externo que articuladas entre sí en un territorio nacional de producción. El caso de la agricultura brasileña, en la que el moderno complejo agrícola del centro-sur tiene poca o ninguna relación con la agricultura de subsistencia del interior de la región Nordeste o de las comunidades rurales de la Amazonia (cuyos patrones de calidad son inferiores), no parece alejarse mucho de esta realidad del Brasil contemporáneo. Obviamente, esto tiene repercusiones en los patrones de calidad de las ocupaciones, como se puede apreciar en el mapa 1.

Como destacan Dos Passos, Bariou y Dubreuil (2006), las inversiones realizadas principalmente en la década de 1970 permitieron la expansión de la frontera agrícola brasileña hacia las regiones Sur y Centro-Oeste, con un notable crecimiento de la productividad agrícola. Así, estas regiones pasaron a desempeñar un papel importante en la economía brasileña, al consolidar un modelo exitoso basado en un sistema agroexportador. La modernización de las actividades agrícolas en estas regiones permitió alcanzar niveles mucho más elevados de productividad que, sumados al nivel de urbanización de estas regiones, permitieron el desarrollo de una masa productiva alineada con la perspectiva de los agronegocios. En términos de calidad de las ocupaciones, este sistema se traduce en niveles de condiciones del trabajo agrícola mucho más altos en relación con las demás regiones brasileñas, como se puede observar en el mapa 1. 


\section{Mapa 1}

Brasil: calidad de las ocupaciones agrícolas rurales y urbanas, por estado, 2014

A. Ocupaciones agrícolas rurales

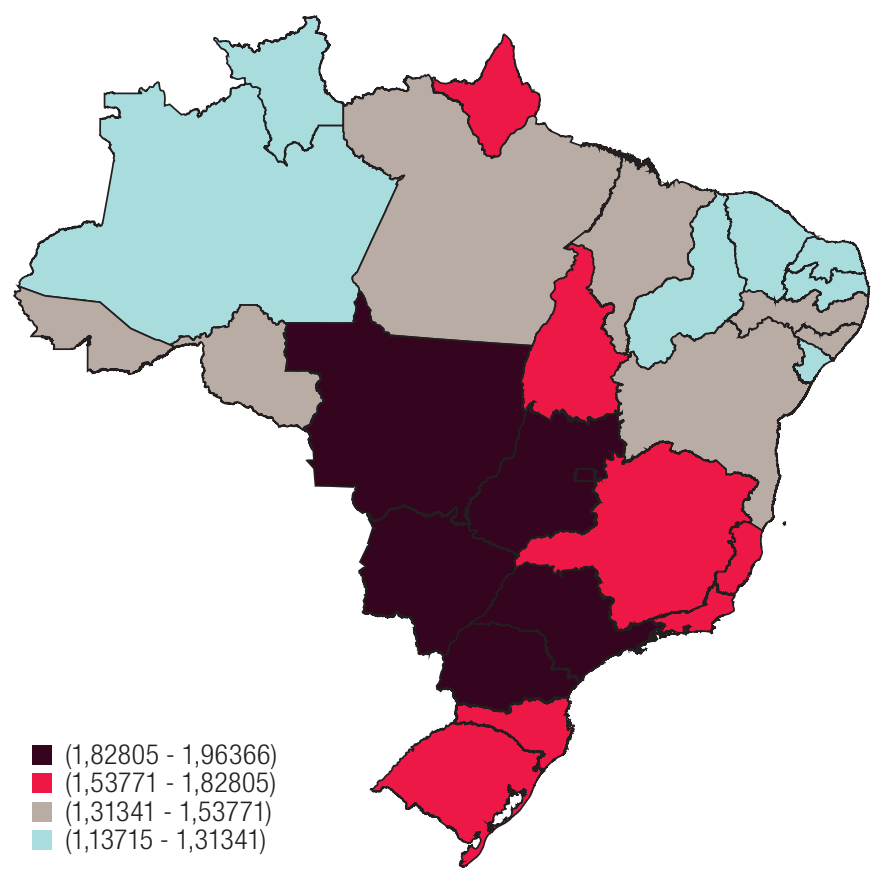

B. Ocupaciones agrícolas urbanas

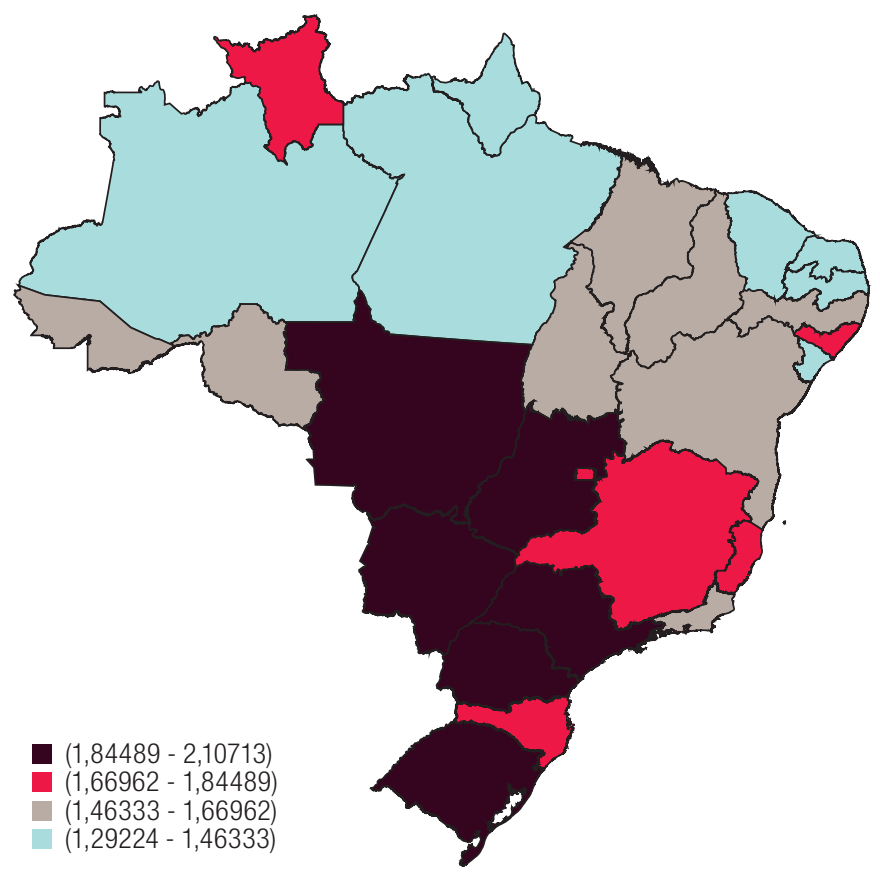

Fuente: Elaboración propia.

Sin embargo, aunque la región Centro-Oeste se destaca en el escenario del empleo agrícola, tiene niveles de calidad menos destacados en términos de empleo no agrícola con respecto a otras regiones del Brasil. No obstante, debido a sus condiciones y características, el empleo no agrícola es 
relativamente mejor que el empleo agrícola. Los estados de las regiones Sur y Sudeste que presentan un mayor desarrollo urbano-industrial son las áreas donde el empleo no agrícola urbano presenta las mejores condiciones, como se puede observar en el mapa 2.

\section{Mapa 2}

Brasil: calidad de las ocupaciones no agrícolas rurales y urbanas, por estado, 2014

A. Ocupaciones no agrícolas rurales

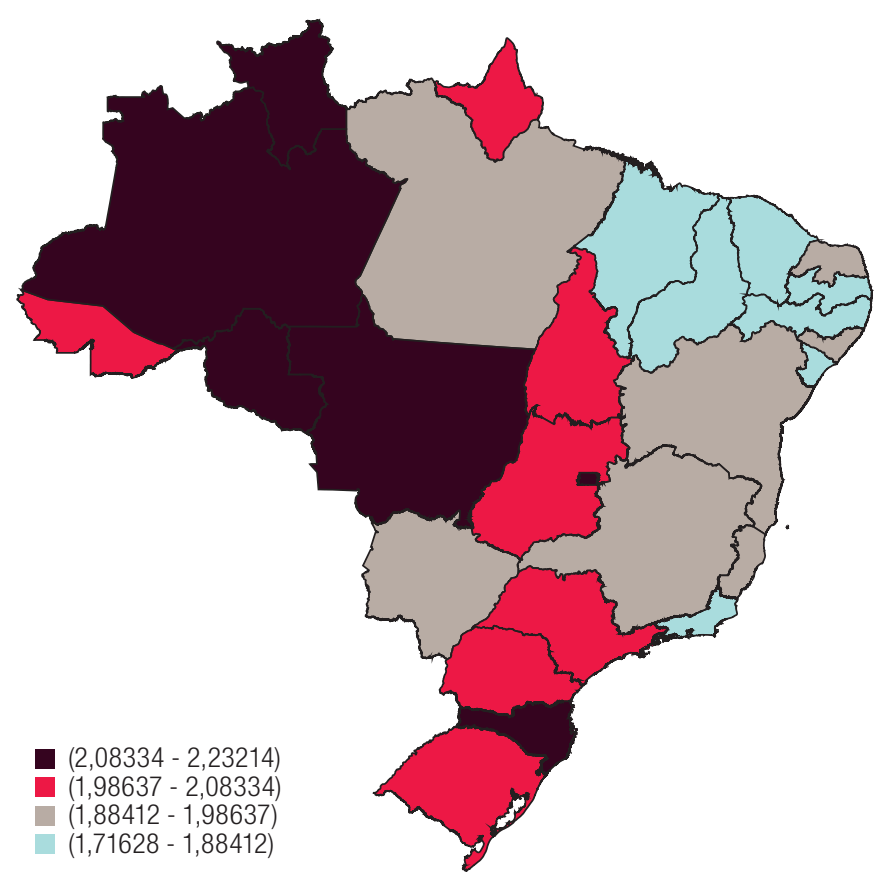

B. Ocupaciones no agrícolas urbanas

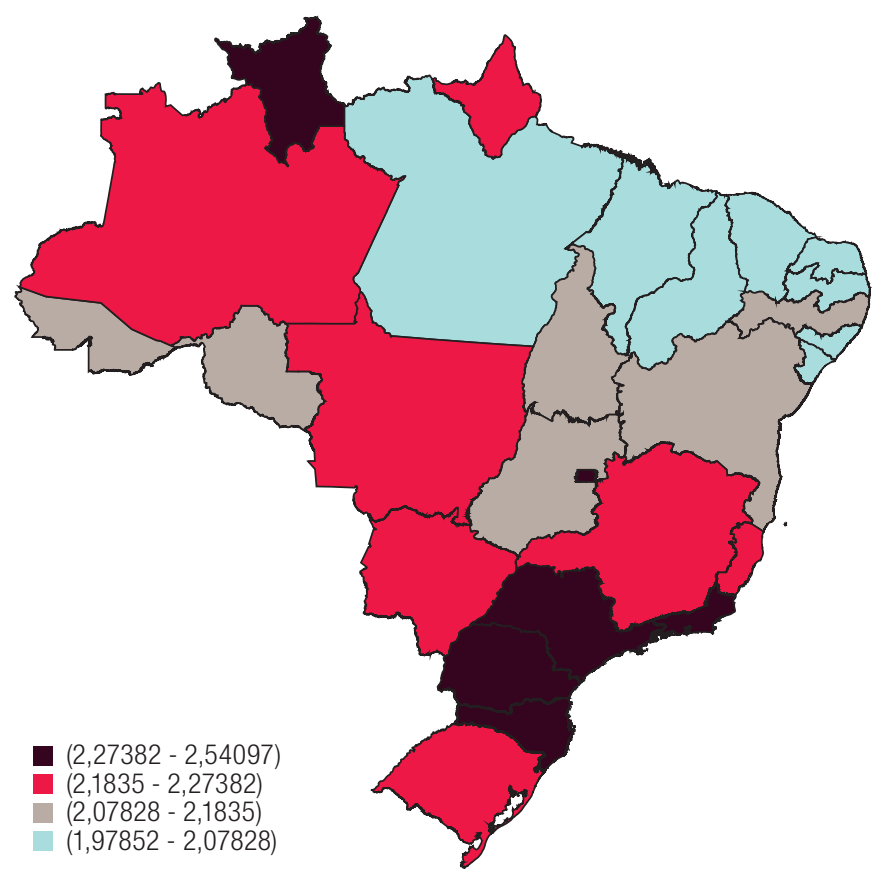

Fuente: Elaboración propia. 
Si bien la región Nordeste presenta algunos avances con respecto al inicio de la serie (véase el cuadro 4), sus resultados son inferiores a los de las demás regiones del país y, en general, se encuentran por debajo de la media nacional. El menor nivel de modernización de las actividades económicas, tanto agrícolas como no agrícolas, determina un contexto relativamente precario en términos de desarrollo y complejidad del mercado de trabajo, que se refleja en el menor nivel de calidad de las ocupaciones en esta región, como se desprende de los mapas 1 y 2. Incluso en los estados del Nordeste donde la actividad económica es más intensa, como en el caso de Bahia, el nivel de desarrollo del mercado de trabajo no es muy alto. Por ese motivo, la región carece de mecanismos que promuevan la mejora de las relaciones de trabajo, pues incluso el empleo no agrícola urbano, en el que generalmente se concentran los mejores patrones de calidad, no se destaca en comparación con las demás regiones.

Cuadro 4

Brasil: índice de calidad del empleo por categoría y unidades subnacionales

\begin{tabular}{|c|c|c|c|c|c|c|c|c|}
\hline & \multicolumn{4}{|c|}{ Rural } & \multicolumn{4}{|c|}{ Urbano } \\
\hline & \multicolumn{2}{|c|}{ Agrícola } & \multicolumn{2}{|c|}{ No agrícola } & \multicolumn{2}{|c|}{ Agrícola } & \multicolumn{2}{|c|}{ No agrícola } \\
\hline & 2004 & 2014 & 2004 & 2014 & 2004 & 2014 & 2004 & 2014 \\
\hline RO & 1,33 & 1,54 & 1,88 & 2,23 & 1,63 & 1,67 & 2,09 & 2,18 \\
\hline AC & 1,32 & 1,41 & 1,80 & 2,00 & 1,29 & 1,53 & 2,12 & 2,15 \\
\hline AM & 1,51 & 1,31 & 1,98 & 2,16 & 1,25 & 1,29 & 2,13 & 2,21 \\
\hline $\mathrm{RR}$ & 1,03 & 1,14 & 1,93 & 2,15 & 1,59 & 1,74 & 2,10 & 2,30 \\
\hline PA & 1,29 & 1,48 & 1,83 & 1,92 & 1,33 & 1,45 & 1,90 & 2,04 \\
\hline AP & 1,17 & 1,65 & 1,76 & 2,08 & 1,48 & 1,46 & 2,14 & 2,27 \\
\hline TO & 1,26 & 1,61 & 1,85 & 2,03 & 1,31 & 1,67 & 1,92 & 2,15 \\
\hline $\mathrm{N}$ & 1,30 & 2,01 & 1,86 & 2,49 & 1,35 & 2,06 & 2,01 & 2,63 \\
\hline MA & 1,09 & 1,37 & 1,56 & 1,88 & 1,22 & 1,62 & 1,76 & 1,99 \\
\hline $\mathrm{PI}$ & 1,00 & 1,22 & 1,66 & 1,84 & 1,09 & 1,48 & 1,83 & 1,98 \\
\hline $\mathrm{CE}$ & 1,02 & 1,26 & 1,47 & 1,80 & 1,03 & 1,41 & 1,86 & 2,02 \\
\hline $\mathrm{RN}$ & 1,14 & 1,28 & 1,75 & 1,92 & 1,14 & 1,44 & 1,96 & 2,08 \\
\hline PB & 1,25 & 1,31 & 1,56 & 1,80 & 1,20 & 1,38 & 1,89 & 2,03 \\
\hline $\mathrm{PE}$ & 1,22 & 1,35 & 1,57 & 1,72 & 1,36 & 1,57 & 1,94 & 2,11 \\
\hline $\mathrm{AL}$ & 1,24 & 1,42 & 1,79 & 1,93 & 1,31 & 1,69 & 1,97 & 2,05 \\
\hline SE & 0,98 & 1,31 & 1,42 & 1,78 & 1,28 & 1,40 & 2,06 & 2,08 \\
\hline $\mathrm{BA}$ & 1,17 & 1,33 & 1,61 & 1,89 & 1,30 & 1,49 & 1,94 & 2,09 \\
\hline $\mathrm{NE}$ & 1,15 & 1,76 & 1,60 & 2,27 & 1,25 & 1,95 & 1,91 & 2,51 \\
\hline$M G$ & 1,36 & 1,62 & 1,66 & 1,95 & 1,52 & 1,79 & 2,05 & 2,19 \\
\hline ES & 1,32 & 1,55 & 1,72 & 1,99 & 1,43 & 1,68 & 2,10 & 2,22 \\
\hline RJ & 1,66 & 1,66 & 1,80 & 1,87 & 1,64 & 1,67 & 2,24 & 2,32 \\
\hline SP & 1,76 & 1,89 & 1,99 & 2,04 & 1,79 & 2,00 & 2,27 & 2,33 \\
\hline SE & 1,46 & 2,21 & 1,85 & 2,48 & 1,66 & 2,38 & 2,21 & 2,80 \\
\hline PR & 1,54 & 1,84 & 1,87 & 2,01 & 1,42 & 1,87 & 2,16 & 2,29 \\
\hline SC & 1,54 & 1,83 & 1,98 & 2,19 & 1,58 & 1,76 & 2,23 & 2,32 \\
\hline $\mathrm{RS}$ & 1,63 & 1,71 & 1,95 & 2,04 & 1,62 & 1,85 & 2,21 & 2,26 \\
\hline S & 1,57 & 2,33 & 1,93 & 2,63 & 1,51 & 2,39 & 2,19 & 2,81 \\
\hline MS & 1,77 & 1,96 & 1,72 & 1,92 & 1,73 & 2,11 & 2,00 & 2,19 \\
\hline MT & 1,76 & 1,93 & 1,88 & 2,11 & 1,61 & 2,07 & 2,04 & 2,21 \\
\hline GO & 1,62 & 1,89 & 1,65 & 2,05 & 1,52 & 1,94 & 1,98 & 2,15 \\
\hline $\mathrm{DF}$ & 1,53 & 1,94 & 2,07 & 2,10 & 2,20 & 1,84 & 2,47 & 2,54 \\
\hline $\mathrm{CO}$ & 1,71 & 2,47 & 1,79 & 2,52 & 1,58 & 2,55 & 2,10 & 2,74 \\
\hline BR & 1,35 & 1,55 & 1,78 & 1,95 & 1,49 & 1,76 & 2,13 & 2,23 \\
\hline
\end{tabular}

Fuente: Elaboración propia.

Los mejores patrones de calidad del empleo agrícola se concentran en las regiones donde el nivel de desarrollo de la agricultura es más elevado. De esta manera, las actividades agrícolas tradicionales, más comunes en la región Nordeste, definen un escenario en el que se establecen relaciones de 
trabajo más precarias que las de la moderna agricultura del centro-sur del país. En los grandes estados productores de granos se registran las mejores tasas de calidad del empleo. En consecuencia, el sistema agroexportador del centro-sur no solo se destaca por su capacidad productiva, sino también por ofrecer mejores relaciones laborales con respecto a las que caracterizan a las ocupaciones esencialmente agrícolas y más tradicionales.

La reducción del empleo agrícola no supuso una disminución significativa de la población rural brasileña en la década de 2000. Esto se debe a que las personas ocupadas en actividades agrícolas buscan puestos de trabajo con mejores condiciones en el mercado de trabajo no agrícola, pero sin desvincularse espacialmente del medio rural (véase el cuadro 3). De esta manera, el empleo no agrícola rural constituye una especie de "intermediario" entre el empleo agrícola rural, de menor calidad, y el empleo no agrícola urbano, de mayor calidad. Los patrones de calidad del empleo no agrícola rural son más altos que los del primero, pero más bajos que los de este último (véase el cuadro 4).

El análisis regional evidencia que los estados de la región Sur, junto con São Paulo, Río de Janeiro y el Distrito Federal, presentan los mejores niveles, especialmente para el empleo no agrícola urbano (véase el mapa 2B). También en esta categoría de empleo, se destaca la presencia de algunos estados del norte del Brasil con un alto índice de calidad, incluso aunque no sean tan dinámicos en términos económicos ni tengan una estructura urbana desarrollada, pues la necesidad de atraer personas de otras partes del país (se trata de las áreas con menor densidad demográfica) se traduce en la concesión de incentivos para los trabajadores que, a su vez, se refleja en una mejor calidad relativa del empleo, como sugieren los resultados de Freguglia, Gonçalves y Da Silva (2014). Este parece ser también el caso del empleo no agrícola rural (véase el mapa 2A), en el que se destaca la región centro-sur. Una vez más, las condiciones de desarrollo de las actividades no agrícolas en estos estados permiten un mejor "entrelazamiento" de las actividades no agrícolas con el medio rural, dado que la dinámica y la magnitud de las actividades económicas que allí se desarrollan producen relaciones de trabajo más avanzadas en comparación con las otras áreas del país.

Los resultados presentados hasta ahora muestran un mercado de trabajo con una gran heterogeneidad y asimetría, tanto en términos sectoriales como espaciales. El complejo económico del centro-sur tiene una estructura laboral mucho más avanzada en términos de calidad con respecto a las regiones Nordeste y Norte, donde los avances se sienten con más fuerza, pero son aún insuficientes para corregir el atraso en relación con las demás regiones brasileñas.

La rápida marcha del Brasil hacia el capitalismo industrial reforzó las características del mercado de trabajo extremadamente asimétrico y desconectado. A principios de la década de 1960, estos paradigmas indujeron una fuerte migración del campo a la ciudad, que culminó con una percepción de vaciamiento de las zonas rurales. La nueva configuración del mercado laboral agrícola y no agrícola, caracterizada por su heterogeneidad, promueve una dinámica migratoria diferente, en términos sectoriales y no espaciales como en el pasado (Fajardo, 2008; Priori y otros, 2012).

Los resultados presentados en los mapas 1 y 2 también ayudan a comprender que las regiones menos conectadas al patrón agroindustrial brasileño perfilan un grupo de trabajadores con una calidad ocupacional más precaria (tanto en términos espaciales como sectoriales), resultado del desempleo y el subempleo crónico como fenómenos específicos de la formación económico-social de estas regiones y del Brasil, que en el pasado se tradujo en intensos flujos migratorios desde las áreas rurales de estas regiones hacia las áreas urbanas del gran complejo agroindustrial del centro-sur y que ahora continúa en el pasaje del sector agrícola al no agrícola. Este flujo migratorio mixto es inherente al modelo de crecimiento económico dual que ha caracterizado la trayectoria de desarrollo del Brasil. Muchos trabajadores están inmersos en un sistema de producción tradicional y precario con baja productividad, que en las regiones más pobres estimula una transferencia de mano de obra a la región o el sector más modernos, donde la productividad del trabajo es mayor y, por lo tanto, mejora la calidad del empleo. En este escenario, cabe destacar la falta de oportunidades de la población rural de las regiones más 
pobres para acceder a la tierra, la resistencia del latifundio a la constitución de una población campesina, y las formas de gestión capitalistas más atrasadas, que tienen repercusiones en el mercado de trabajo.

Cabe señalar que, a lo largo del período examinado, se registraron mejoras significativas en la calidad de las ocupaciones, especialmente en las regiones donde esta es más baja. Sin embargo, como demuestran los datos del cuadro 4, todavía persisten importantes heterogeneidades, tanto regionales como sectoriales. Entre otras cosas, el empleo en las regiones Norte y Nordeste es relativamente de peor calidad que el empleo en el complejo agroindustrial del centro-sur del país y el empleo agrícola presenta peores patrones de calidad con respecto al empleo no agrícola.

\section{IV. Índice de calidad del empleo: resultados empíricos}

En esta sección se detallan y analizan los resultados de los modelos econométricos presentados en la sección de aspectos metodológicos, que relacionan el índice de calidad del empleo para los cuatro grupos estudiados con arreglo a un conjunto de variables que sintetizan algunos de los condicionantes de la estructura del mercado de trabajo brasileño. En consecuencia, esta sección se organiza según la interpretación de cuatro cuadros referidos a las estimaciones de las cuatro ecuaciones propuestas en dicha sección (véanse los cuadros 5, 6, 7 y 8). La estructura de interpretación comienza con los datos sobre el empleo agrícola rural, seguido por el empleo agrícola urbano, el empleo no agrícola rural y, por último, el empleo no agrícola urbano.

\section{Cuadro 5}

Resultados de las estimaciones de los modelos seleccionados para el mercado de trabajo agrícola rural

\begin{tabular}{|c|c|c|c|c|c|c|c|c|c|c|c|c|}
\hline \multirow[t]{2}{*}{ Variables } & \multicolumn{3}{|c|}{$\begin{array}{l}\text { Mínimos cuadrados } \\
\text { ordinarios } \\
{[\mathrm{A}]}\end{array}$} & \multicolumn{3}{|c|}{$\begin{array}{l}\text { Panel de efectos fijos } \\
\text { [B] }\end{array}$} & \multicolumn{3}{|c|}{$\begin{array}{c}\text { Regresión de variables } \\
\text { instrumentales } \\
{[\mathrm{C}]} \\
\end{array}$} & \multicolumn{3}{|c|}{$\begin{array}{l}\text { Método generalizado } \\
\text { de momentos } \\
{[D]}\end{array}$} \\
\hline & Coef & $t$ & $p>|t|$ & Coef & $t$ & $p>|t|$ & Coef & z & $p>|z|$ & Coef & z & $p>|z|$ \\
\hline $\begin{array}{l}\text { Índice de calidad } \\
\text { del empleo }\end{array}$ & 0,721 & 12,39 & 0,000 & 0,140 & 1,98 & 0,049 & 0,494 & 4,28 & 0,000 & 0,494 & 4,28 & 0,000 \\
\hline $\begin{array}{l}\text { Valor agregado } \\
\text { agrícola }\end{array}$ & 0,006 & 0,87 & 0,387 & $-0,046$ & $-1,95$ & 0,053 & 0,104 & 2,52 & 0,012 & 0,104 & 2,52 & 0,012 \\
\hline $\begin{array}{l}\text { Valor agregado } \\
\text { no agrícola }\end{array}$ & $-0,009$ & $-1,50$ & 0,135 & 0,139 & 3,43 & 0,001 & $-0,082$ & $-2,34$ & 0,019 & $-0,082$ & $-2,34$ & 0,019 \\
\hline Ingreso & 0,047 & 3,05 & 0,003 & 0,034 & 1,51 & 0,132 & 0,095 & 2,80 & 0,005 & 0,095 & 2,80 & 0,005 \\
\hline Desigualdad & 0,090 & 0,93 & 0,353 & $-0,050$ & $-0,42$ & 0,676 & 0,208 & 0,55 & 0,583 & 0,208 & 0,55 & 0,583 \\
\hline Educación & 0,028 & 1,82 & 0,071 & $-0,127$ & $-2,52$ & 0,013 & 0,093 & 2,12 & 0,034 & 0,093 & 2,12 & 0.034 \\
\hline \multirow{10}{*}{$\begin{array}{l}\text { Pruebas } \\
\text { estadísticas }\end{array}$} & \multicolumn{3}{|c|}{$F(6 ; 210)=1860,37$} & \multicolumn{3}{|c|}{$F(6 ; 183)=16,06$} & \multicolumn{3}{|c|}{$F(6 ; 7)=2071,99$} & \multicolumn{3}{|c|}{ wald(6) $=14546,24$} \\
\hline & \multicolumn{3}{|c|}{ Prob $>F=0,000$} & \multicolumn{3}{|c|}{ Prob $>F=0,000$} & \multicolumn{3}{|c|}{ Prob $>F=0,000$} & \multicolumn{3}{|c|}{ Prob $>$ chi2 $=0,000$} \\
\hline & \multicolumn{3}{|c|}{$\begin{array}{c}\text { Número de } \\
\text { observaciones }=216\end{array}$} & \multicolumn{3}{|c|}{$\begin{array}{c}\text { Número de } \\
\text { observaciones }=216\end{array}$} & \multicolumn{3}{|c|}{$\begin{array}{c}\text { Número de } \\
\text { observaciones }=216\end{array}$} & \multicolumn{3}{|c|}{$\begin{array}{c}\text { Número de } \\
\text { observaciones }=216\end{array}$} \\
\hline & \multicolumn{3}{|c|}{$\mathrm{R}^{2}$ ajustado $=0,970$} & \multicolumn{3}{|c|}{$\begin{array}{c}\mathrm{R}^{2} \text { dentro } \\
\text { (within) }=0,3449\end{array}$} & \multicolumn{3}{|c|}{$\begin{array}{c}\mathrm{R}^{2} \text { no centrado } \\
\text { (uncentered) }=0,93\end{array}$} & & & \\
\hline & & & & \multicolumn{3}{|c|}{ Grupos = 27} & \multicolumn{3}{|c|}{ Grupos $=8$} & \multicolumn{3}{|c|}{ Grupos $=27$} \\
\hline & & & & & & & \multicolumn{3}{|c|}{ Kleibergen $=0,0158$} & \multicolumn{3}{|c|}{ Instrumentos $=7$} \\
\hline & \multicolumn{9}{|c|}{ H0: Ausencia de autocorrelación de primer orden } & \multicolumn{3}{|c|}{0,039} \\
\hline & \multicolumn{9}{|c|}{ HO: Ausencia de autocorrelación de segundo orden } & \multicolumn{3}{|c|}{0,260} \\
\hline & \multicolumn{9}{|c|}{ Prueba de Sargan } & \multicolumn{3}{|c|}{0,608} \\
\hline & \multicolumn{9}{|c|}{ Prueba de Hansen } & \multicolumn{3}{|c|}{0,621} \\
\hline
\end{tabular}

Fuente: Elaboración propia. 


\section{Cuadro 6}

Resultados de las estimaciones de los modelos seleccionados

para el mercado de trabajo agrícola urbano

\begin{tabular}{|c|c|c|c|c|c|c|c|c|c|c|c|c|}
\hline \multirow[t]{2}{*}{ Variables } & \multicolumn{3}{|c|}{$\begin{array}{c}\text { Mínimos cuadrados } \\
\text { ordinarios } \\
{[\mathrm{A}]} \\
\end{array}$} & \multicolumn{3}{|c|}{$\begin{array}{l}\text { Panel de efectos fijos } \\
{[\text { [B] }}\end{array}$} & \multicolumn{3}{|c|}{$\begin{array}{c}\text { Regresión de variables } \\
\text { instrumentales } \\
{[C]} \\
\end{array}$} & \multicolumn{3}{|c|}{$\begin{array}{l}\text { Método generalizado } \\
\text { de momentos } \\
\text { [D] }\end{array}$} \\
\hline & Coef & $t$ & $p>|t|$ & Coef & $t$ & $p>|t|$ & Coef & $z$ & $p>|z|$ & Coef & $z$ & $p>|z|$ \\
\hline $\begin{array}{l}\text { Índice de calidad } \\
\text { del empleo }\end{array}$ & 0,599 & 8,7 & 0,000 & 0,109 & 1,5 & 0,137 & 0,562 & 7,4 & 0,000 & 0,562 & 7,4 & 0,000 \\
\hline $\begin{array}{l}\text { Valor agregado } \\
\text { agrícola }\end{array}$ & 0,018 & 2,5 & 0,013 & 0,016 & 0,6 & 0,551 & 0,027 & 3,1 & 0,002 & 0,027 & 3,1 & 0,002 \\
\hline $\begin{array}{l}\text { Valor agregado } \\
\text { no agrícola }\end{array}$ & $-0,005$ & $-0,9$ & 0,392 & 0,072 & 1,5 & 0,126 & $-0,003$ & $-0,9$ & 0,348 & $-0,003$ & $-0,9$ & 0,348 \\
\hline Ingreso & 0,032 & 2,6 & 0,010 & 0,042 & 2,2 & 0,031 & 0,066 & 4,9 & 0,000 & 0,066 & 4,9 & 0,000 \\
\hline Desigualdad & $-0,028$ & $-0,3$ & 0,773 & $-0,279$ & $-2,0$ & 0,045 & 0,452 & 2,5 & 0,011 & 0,452 & 2,5 & 0,011 \\
\hline Educación & 0,079 & 4,0 & 0,000 & $-0,044$ & $-0,8$ & 0,454 & 0,048 & 2,4 & 0,016 & 0,048 & 2,4 & 0,016 \\
\hline \multirow{10}{*}{$\begin{array}{l}\text { Pruebas } \\
\text { estadísticas }\end{array}$} & \multicolumn{3}{|c|}{$F(6 ; 210)=2050,09$} & \multicolumn{3}{|c|}{$F(6 ; 183)=26,08$} & \multicolumn{3}{|c|}{$F(6 ; 26)=1039,96$} & \multicolumn{3}{|c|}{ wald $(6)=6634,06$} \\
\hline & \multicolumn{3}{|c|}{ Prob $>F=0,000$} & \multicolumn{3}{|c|}{ Prob $>F=0,000$} & \multicolumn{3}{|c|}{ Prob $>F=0,000$} & \multicolumn{3}{|c|}{ Prob $>$ chi2 $=0,000$} \\
\hline & \multicolumn{3}{|c|}{$\begin{array}{c}\text { Número de } \\
\text { observaciones = } 216\end{array}$} & \multicolumn{3}{|c|}{$\begin{array}{c}\text { Número de } \\
\text { observaciones }=216\end{array}$} & \multicolumn{3}{|c|}{$\begin{array}{c}\text { Número de } \\
\text { observaciones = } 216\end{array}$} & \multicolumn{3}{|c|}{$\begin{array}{c}\text { Número de } \\
\text { observaciones = } 216\end{array}$} \\
\hline & \multicolumn{3}{|c|}{$\mathrm{R}^{2}$ ajustado $=0,9696$} & \multicolumn{3}{|c|}{$\begin{array}{c}\mathrm{R}^{2} \text { dentro } \\
\text { (within) }=0,4609\end{array}$} & \multicolumn{3}{|c|}{$\begin{array}{c}\mathrm{R}^{2} \text { no centrado } \\
\text { (uncentered) }=0,97\end{array}$} & & & \\
\hline & & & & \multicolumn{3}{|c|}{ Grupos = 27} & \multicolumn{3}{|c|}{ Grupos $=27$} & \multicolumn{3}{|c|}{ Grupos $=27$} \\
\hline & & & & & & & \multicolumn{3}{|c|}{ Kleibergen $=0,0071$} & \multicolumn{3}{|c|}{ Instrumentos $=7$} \\
\hline & \multicolumn{9}{|c|}{ H0: Ausencia de autocorrelación de primer orden } & \multicolumn{3}{|c|}{0,001} \\
\hline & \multicolumn{9}{|c|}{ HO: Ausencia de autocorrelación de segundo orden } & \multicolumn{3}{|c|}{0,523} \\
\hline & \multicolumn{9}{|c|}{ Prueba de Sargan } & \multicolumn{3}{|c|}{0,394} \\
\hline & \multicolumn{9}{|c|}{ Prueba de Hansen } & \multicolumn{3}{|c|}{0,386} \\
\hline
\end{tabular}

Fuente: Elaboración propia.

\section{Cuadro 7}

Resultados de las estimaciones de los modelos seleccionados para el mercado de trabajo no agrícola rural

\begin{tabular}{|c|c|c|c|c|c|c|c|c|c|c|c|c|}
\hline \multirow[t]{2}{*}{ Variables } & \multicolumn{3}{|c|}{$\begin{array}{l}\text { Mínimos cuadrados } \\
\text { ordinarios } \\
{[\mathrm{A}]}\end{array}$} & \multicolumn{3}{|c|}{$\begin{array}{l}\text { Panel de efectos fijos } \\
\text { [B] }\end{array}$} & \multicolumn{3}{|c|}{$\begin{array}{l}\text { Regresión de variables } \\
\text { instrumentales } \\
\text { [C] }\end{array}$} & \multicolumn{3}{|c|}{$\begin{array}{c}\text { Método generalizado } \\
\text { de momentos } \\
\text { [D] }\end{array}$} \\
\hline & Coef & $t$ & $\mathrm{p}>|\mathrm{t}|$ & Coef & $\mathrm{t}$ & $p>|t|$ & Coef & z & $p>|z|$ & Coef & $z$ & $p>|z|$ \\
\hline $\begin{array}{l}\text { Índice de calidad } \\
\text { del empleo }\end{array}$ & 0,465 & 8,0 & 0,000 & 0,071 & 1,2 & 0,253 & 0,405 & 5,0 & 0,000 & 0,405 & 5,0 & 0,000 \\
\hline $\begin{array}{l}\text { Valor agregado } \\
\text { agrícola }\end{array}$ & 0,008 & 1,8 & 0,082 & 0,003 & 0,2 & 0,836 & 0,011 & 1,3 & 0,206 & 0,011 & 1,3 & 0,206 \\
\hline $\begin{array}{l}\text { Valor agregado } \\
\text { no agrícola }\end{array}$ & $-0,011$ & $-3,3$ & 0,001 & $-0,064$ & $-2,5$ & 0,014 & $-0,015$ & $-2,1$ & 0,040 & $-0,015$ & $-2,1$ & 0,040 \\
\hline Ingreso & 0,077 & 8,0 & 0,000 & 0,167 & 8,5 & 0,000 & 0,052 & 6,3 & 0,000 & 0,052 & 6,3 & 0,000 \\
\hline Desigualdad & 0,081 & 1,3 & 0,195 & 0,133 & 1,9 & 0,065 & $-0,292$ & $-2,2$ & 0,029 & $-0,292$ & $-2,2$ & 0,029 \\
\hline Educación & 0,029 & 3,6 & 0,000 & 0,012 & 0,4 & 0,687 & 0,053 & 2,9 & 0,003 & 0,053 & 2,9 & 0,003 \\
\hline \multirow{10}{*}{$\begin{array}{l}\text { Pruebas } \\
\text { estadísticas }\end{array}$} & \multicolumn{3}{|c|}{$F(6 ; 210)=12234,51$} & \multicolumn{3}{|c|}{$F(6 ; 183)=46,10$} & \multicolumn{3}{|c|}{$F(6 ; 7)=3268,60$} & \multicolumn{3}{|c|}{ wald(6) = 22 946,87 } \\
\hline & \multicolumn{3}{|c|}{ Prob $>F=0,000$} & \multicolumn{3}{|c|}{ Prob $>F=0,000$} & \multicolumn{3}{|c|}{ Prob $>F=0,000$} & \multicolumn{3}{|c|}{ Prob $>$ chi2 $=0,000$} \\
\hline & \multicolumn{3}{|c|}{$\begin{array}{c}\text { Número de } \\
\text { observaciones }=216\end{array}$} & \multicolumn{3}{|c|}{$\begin{array}{c}\text { Número de } \\
\text { observaciones }=216\end{array}$} & \multicolumn{3}{|c|}{$\begin{array}{c}\text { Número de } \\
\text { observaciones }=216\end{array}$} & \multicolumn{3}{|c|}{$\begin{array}{c}\text { Número de } \\
\text { observaciones }=216\end{array}$} \\
\hline & \multicolumn{3}{|c|}{$R^{2}$ ajustado $=0,9957$} & \multicolumn{3}{|c|}{$\begin{array}{c}\mathrm{R}^{2} \text { dentro } \\
\text { (within) }=0,6018\end{array}$} & \multicolumn{3}{|c|}{$\begin{array}{c}\mathrm{R}^{2} \text { no centrado } \\
\text { (uncentered) }=0,995\end{array}$} & & & \\
\hline & & & & \multicolumn{3}{|c|}{ Grupos = 27} & \multicolumn{3}{|c|}{ Grupos $=8$} & \multicolumn{3}{|c|}{ Grupos = 27} \\
\hline & & & & & & & \multicolumn{3}{|c|}{ Kleibergen $=0,0033$} & \multicolumn{3}{|c|}{ Instrumentos $=7$} \\
\hline & \multicolumn{9}{|c|}{ HO: Ausencia de autocorrelación de primer orden } & \multicolumn{3}{|c|}{0,004} \\
\hline & \multicolumn{9}{|c|}{ H0: Ausencia de autocorrelación de segundo orden } & \multicolumn{3}{|c|}{0,561} \\
\hline & \multicolumn{9}{|c|}{ Prueba de Sargan } & \multicolumn{3}{|c|}{0,819} \\
\hline & \multicolumn{9}{|c|}{ Prueba de Hansen } & \multicolumn{3}{|c|}{0,562} \\
\hline
\end{tabular}

Fuente: Elaboración propia. 


\section{Cuadro 8}

Resultados de las estimaciones de los modelos seleccionados para el mercado de trabajo no agrícola urbano

\begin{tabular}{|c|c|c|c|c|c|c|c|c|c|c|c|c|}
\hline \multirow[t]{2}{*}{ Variables } & \multicolumn{3}{|c|}{$\begin{array}{c}\text { Mínimos cuadrados } \\
\text { ordinarios } \\
{[\mathrm{A}]}\end{array}$} & \multicolumn{3}{|c|}{$\begin{array}{l}\text { Panel de efectos fijos } \\
{[B]}\end{array}$} & \multicolumn{3}{|c|}{$\begin{array}{l}\text { Regresión de variables } \\
\text { instrumentales } \\
{[\mathrm{C}]}\end{array}$} & \multicolumn{3}{|c|}{$\begin{array}{c}\text { Método generalizado } \\
\text { de momentos } \\
{[\mathrm{D}]}\end{array}$} \\
\hline & Coef & $\mathrm{T}$ & $p>|t|$ & Coef & $\mathrm{t}$ & $p>|t|$ & Coef & $z$ & $\mathrm{p}>|\mathrm{z}|$ & Coef & z & $p>|z|$ \\
\hline $\begin{array}{l}\text { Índice de calidad } \\
\text { del empleo }\end{array}$ & 0,849 & 29,6 & 0,000 & 0,407 & 8,0 & 0,000 & 0,834 & 15,3 & 0,000 & 0,834 & 15,3 & 0,000 \\
\hline $\begin{array}{l}\text { Valor agregado } \\
\text { agrícola }\end{array}$ & $-0,001$ & $-0,8$ & 0,415 & 0,004 & 0,8 & 0,406 & $-0,003$ & $-1,3$ & 0,196 & $-0,003$ & $-1,3$ & 0,196 \\
\hline $\begin{array}{l}\text { Valor agregado } \\
\text { no agrícola }\end{array}$ & 0,001 & 0,5 & 0,649 & $-0,079$ & $-4,6$ & 0,000 & 0,002 & 1,5 & 0,138 & 0,002 & 1,5 & 0,138 \\
\hline Ingreso & 0,020 & 5,5 & 0,000 & 0,105 & 5,3 & 0,000 & 0,018 & 3,0 & 0,002 & 0,018 & 3,0 & 0,002 \\
\hline Desigualdad & 0,012 & 0,8 & 0,454 & $-0,080$ & $-2,8$ & 0,007 & $-0,028$ & $-0,7$ & 0,477 & $-0,028$ & $-0,7$ & 0,477 \\
\hline Educación & 0,0 & 2,3 & 0,023 & 0,051 & 5,2 & 0,000 & 0,007 & 1,6 & 0,100 & 0,007 & 1,6 & 0,100 \\
\hline \multirow{10}{*}{$\begin{array}{l}\text { Pruebas } \\
\text { estadísticas }\end{array}$} & \multicolumn{3}{|c|}{$F(6 ; 210)=99999$} & \multicolumn{3}{|c|}{$F(6 ; 183)=167,93$} & \multicolumn{3}{|c|}{$F(6 ; 7)=64182,17$} & \multicolumn{3}{|c|}{ wald(6) = 450585} \\
\hline & \multicolumn{3}{|c|}{ Prob $>F=0,000$} & \multicolumn{3}{|c|}{ Prob $>F=0,000$} & \multicolumn{3}{|c|}{ Prob $>F=0,000$} & \multicolumn{3}{|c|}{ Prob $>$ chi2 $=0,00$} \\
\hline & \multicolumn{3}{|c|}{$\begin{array}{c}\text { Número de } \\
\text { observaciones = } 216\end{array}$} & \multicolumn{3}{|c|}{$\begin{array}{c}\text { Número de } \\
\text { observaciones }=216\end{array}$} & \multicolumn{3}{|c|}{$\begin{array}{c}\text { Número de } \\
\text { observaciones = } 216\end{array}$} & \multicolumn{3}{|c|}{$\begin{array}{c}\text { Número de } \\
\text { observaciones }=216\end{array}$} \\
\hline & \multicolumn{3}{|c|}{$\mathrm{R}^{2}$ ajustado $=0,9997$} & \multicolumn{3}{|c|}{$\begin{array}{c}\mathrm{R}^{2} \text { dentro } \\
\text { (within) }=0,8463\end{array}$} & \multicolumn{3}{|c|}{$\begin{array}{c}\mathrm{R}^{2} \text { no centrado } \\
\text { (uncentered) }=0,9997\end{array}$} & & & \\
\hline & & & & \multicolumn{3}{|c|}{ Grupos = 27} & \multicolumn{3}{|c|}{ Grupos $=8$} & \multicolumn{3}{|c|}{ Grupos $=27$} \\
\hline & & & & & & & \multicolumn{3}{|c|}{ Kleibergen $=0,0000$} & \multicolumn{3}{|c|}{ Instrumentos $=9$} \\
\hline & \multicolumn{9}{|c|}{ HO: Ausencia de autocorrelación de primer orden } & \multicolumn{3}{|c|}{0,000} \\
\hline & \multicolumn{9}{|c|}{ HO: Ausencia de autocorrelación de segundo orden } & \multicolumn{3}{|c|}{0,979} \\
\hline & \multicolumn{9}{|c|}{ Prueba de Sargan } & \multicolumn{3}{|c|}{0,189} \\
\hline & \multicolumn{9}{|c|}{ Prueba de Hansen } & \multicolumn{3}{|c|}{0,998} \\
\hline
\end{tabular}

Fuente: Elaboración propia.

En la columna $[\mathrm{A}]$ de cada cuadro se presenta el resultado de las estimaciones por mínimos cuadrados ordinarios y en la columna [B] las estimaciones del método de panel de efectos fijos, que en este modelo resulta más eficiente con respecto al método de panel de efectos aleatorios, según la prueba de Hausman en los cuatro modelos estimados. En la columna [C] se presenta el modelo con variables instrumentales, utilizado para evaluar la posibilidad de subidentificación. Por último, en la columna [D] se presentan las estimaciones de los parámetros por medio del método generalizado de momentos para sistemas (MGM-sistema). Cabe observar que [C] y [D] son, esencialmente, el mismo modelo. Como se mencionó anteriormente, el MGM-sistema es el resultado de una extensión del estimador original de Arellano y Bond (1991), propuesta por Arellano y Bover (1995) y desarrollada por Blundell y Bond (1998).

Como se observa en la columna [A] de cada cuadro, los valores de los coeficientes estimados de la variable "calidad del empleo rezagada en un período" por el método de mínimos cuadrados ordinarios son, de hecho, superiores a los valores estimados en la columna [B] para esta misma variable por el método de datos de panel de efectos fijos. Si los instrumentos utilizados son adecuados, el valor del coeficiente para esta variable estimado por el método generalizado de momentos debería situarse entre los límites de los coeficientes estimados por los dos métodos anteriores. Esto es precisamente lo que ocurre en la columna [D]. Satisfecha esta característica, el sesgo causado por la presencia de variables endógenas en el lado derecho de la regresión y los efectos fijos no observables se corrigieron mediante el método generalizado de momentos.

De este modo, entre los modelos presentados en los cuadros 5, 6, 7 y 8, se optó siempre por los indicados en la columna [D] de cada cuadro. Las pruebas realizadas en el método generalizado de momentos muestran que las propiedades estadísticas de los modelos son aceptables. La prueba de Hansen, que indica si los instrumentos utilizados son válidos, y la prueba de Sargan, que verifica la 
validez de los instrumentos adicionales requeridos por el MGM-sistema, se satisfacen para los cuatro modelos estimados, como se puede verificar en los cuadros 5, 6, 7 y 8. Asimismo, cabe notar que se rechaza la hipótesis nula de la prueba de subidentificación de Kleibergen y Paap (2006), lo que indica que el modelo contenido en la columna [C] no está subidentificado ${ }^{1}$. Obsérvese también que, si bien las estimaciones de $[\mathrm{C}]$ y $[\mathrm{D}]$ son diferentes, se trata básicamente del mismo modelo, lo que indica que [D] tampoco está subidentificado.

Por último, se incluyen también las pruebas estadísticas de Arellano y Bond (1991) para evaluar la existencia de autocorrelación tanto de primer como de segundo orden. Se observa que la ausencia de autocorrelación de segundo orden es esencial para la consistencia del estimador del método generalizado de momentos. La prueba confirma que no se rechaza la autocorrelación de primer orden, no obstante se rechace la hipótesis de autocorrelación de segundo orden en los cuatro modelos presentados, como se esperaba y conforme las instrucciones en la literatura sobre datos de panel. Por lo tanto, los modelos se califican como aceptables.

En primer lugar, se observa que el coeficiente de la variable "valor agregado agrícola" no es estadísticamente significativo para el empleo no agrícola, ya sea urbano o rural. Esto muestra un comportamiento importante del mercado de trabajo no agrícola según los grupos estudiados. El crecimiento de la producción agrícola no causa, en principio, cambios estructurales en el mercado de trabajo no agrícola. Lo que se capta aquí es el bajo efecto de encadenamiento del producto agrícola en el mercado de trabajo general pues, en promedio, el sector representó solo el 5,7\% del producto total de la economía brasileña en el período de referencia. Aunque el crecimiento de la producción agrícola puede tener algún efecto de encadenamiento en la producción no agrícola y, por lo tanto, en el nivel de empleo, el simple incremento del número de trabajadores no implica un aumento de la calidad de las ocupaciones. Además, el coeficiente de la variable "valor agregado no agrícola" tampoco es significativo para el empleo no agrícola urbano. Así, el crecimiento del mercado de trabajo no agrícola urbano inducido por el crecimiento de la producción agrícola o no agrícola, no garantiza, por sí solo, la modificación de las relaciones de trabajo existentes o nuevas. Así, la calidad de estas nuevas contrataciones es, en promedio, similar a la calidad del empleo de aquellos que ya participan en el mercado, sin que se modifique la estructura del trabajo en estos grupos.

Sin embargo, al examinar los niveles de significación de la variable "valor agregado agrícola" para los grupos de trabajo agrícola - rural (véase el cuadro 5) y urbano (véase el cuadro 6) - se observa que son positivos y estadísticamente significativos a un nivel de significación de por lo menos el $5 \%$. Esto muestra que el comportamiento del empleo agrícola responde de manera más sensible al crecimiento de la producción agrícola que el empleo no agrícola. Así, el aumento del dinamismo económico de la agricultura tiene efectos significativos en la calidad del empleo agrícola.

El signo negativo del valor agregado no agrícola indica que el crecimiento de las actividades industriales y de servicios tiene una relación inversa con la calidad del empleo para los grupos en los que esta variable tiene signo negativo (empleo agrícola rural, agrícola urbano ${ }^{2}$ y no agrícola rural3). De

\footnotetext{
1 La prueba de subidentificación sirve para determinar si la ecuación está identificada, es decir, si los instrumentos excluidos son "relevantes", o sea, si están correlacionados con los regresores endógenos. Se trata esencialmente de la prueba de rango de una matriz: bajo la hipótesis nula de que la ecuación está subidentificada, la matriz de coeficientes de forma reducida en los instrumentos excluidos de L1 tiene rango $=\mathrm{K} 1-1$ donde $\mathrm{K} 1=$ número de regresores endógenos. Bajo la hipótesis nula, la estadística se distribuye como chi-cuadrado con grados de libertad $=(L 1-K 1+1)$. El rechazo de la hipótesis nula indica que la matriz tiene el rango de columna completo, es decir, el modelo está identificado. Véase más información en Kleibergen y Paap (2006).

2 Siguiendo la línea de razonamiento desarrollada en este párrafo, el empleo agrícola urbano debe insertarse con precaución. Si bien el signo del coeficiente del valor agregado no agrícola es negativo como el de los demás tipos de empleo, es débilmente no significativo, de manera que se recomienda cautela con este resultado y las inferencias basadas en él.

3 Aunque el empleo no agrícola rural es un "producto" de la migración sectorial de los trabajadores de las actividades agrícolas a las no agrícolas, todavía parece haber una fuerte influencia de la agricultura en el mercado de trabajo para estos trabajadores, pues estos permanecen vinculados espacialmente a lo rural y, por tanto, a la agricultura. Así, su dinámica, cuando es inducida por las actividades no agrícolas, todavía presenta similitudes con el comportamiento de las actividades puramente agrícolas.
} 
acuerdo con Balsadi y Delgrossi (2018), el proceso de "envejecimiento" de la población ocupada en la agricultura es progresivo y el abandono de las actividades agrícolas - en mayor medida por las mujeres y los jóvenes - contribuye a la precarización de este tipo de empleo e incluso a la discontinuidad de las empresas agrícolas, como se ha observado para algunas regiones del Brasil. Así, el crecimiento de la producción no agrícola, que ofrece nuevas oportunidades de empleo a estos grupos, contribuye al desempeño relativamente menos dinámico del empleo agrícola, con repercusiones en su calidad, como evidencian los modelos econométricos.

Asimismo, se observa que las formas de contratación de la mano de obra en las actividades no agrícolas tienen un nivel de formalidad mucho más alto, que se traduce en mejores ingresos, menos horas de trabajo y menor incidencia del trabajo infantil. De esta manera, a medida que las actividades se desarrollan, tienden a influir en el trabajo agrícola mediante el proceso de migración sectorial, pues los trabajadores agrícolas procuran mejorar su situación dedicándose a actividades en otros sectores donde los niveles de calidad son más altos.

El coeficiente de la variable "ingreso", que expresa el ingreso medio del trabajo para cada unidad de la federación, es estadísticamente significativo a un nivel de significación de por lo menos el 5\% y, como se esperaba, tiene signo positivo para las cuatro ecuaciones analizadas. Así, el incremento del ingreso medio del trabajo tiene importantes efectos en la calidad del empleo. Esto indica que una política de valorización de los ingresos reales del trabajo puede ser importante para mejorar los patrones de calidad de las ocupaciones y, sobre todo, el perfil de los trabajadores. Desde esta perspectiva, el aumento del ingreso está condicionado a puestos de trabajo con mejores formas de contratación de la mano de obra. También se puede pensar que el aumento del ingreso induce un proceso de competencia dentro del mercado de trabajo. Quienes ofrecen empleo se ven incentivados a mejorar sus formas de contratación para conseguir los trabajadores que desean y los trabajadores, a su vez, se sienten menos incentivados a permanecer en puestos de trabajo precarios (de calidad inferior), con niveles de ingreso más bajos, por lo menos en un ambiente de crecimiento económico y disminución del desempleo, como el que se observa en la mayor parte del período analizado.

Con respecto a las relaciones de elasticidad, se observa que la elasticidad calidad-ingreso del empleo es inferior a la unidad en todos los casos, por lo tanto, más inelástica. Aún así, la idea de valorización del ingreso medio del trabajo en cada unidad de la federación puede contribuir a reducir las diferencias entre los mercados de trabajo, tanto urbano y rural como no agrícola y agrícola, ya que los efectos del incremento del nivel de ingreso estatal son menores en los primeros, como se puede observar en los coeficientes de los cuadros 5, 6, 7 y 8.

La variable "desigualdad", expresada en términos del índice de Gini, que mide el nivel de concentración del ingreso, es estadísticamente significativa y negativa para el empleo no agrícola rural y negativa, pero no significativa, para el empleo no agrícola urbano. En este caso, la estructura de concentración del ingreso influye en el nivel de calidad del trabajo en este segmento del mercado laboral. La reducción del índice de Gini implica que el nivel de ingresos de los trabajadores más pobres converge a un nivel de ingresos más elevado, con sus respectivas repercusiones en términos de calidad del empleo, pues la variable "desigualdad" es inversamente proporcional al índice de calidad del empleo. Sin embargo, en el caso del empleo no agrícola urbano, el índice de Gini no parece influir significativamente en la calidad del empleo. Así, las relaciones de trabajo no agrícola urbano mantienen una dinámica propia, independientemente del nivel de concentración del ingreso dado.

Por último, la variable "educación", que expresa la población que ha completado al menos la educación secundaria en cada estado, es significativa a un nivel de significación de por lo menos el $10 \%$ y tiene signo positivo, según el comportamiento esperado dentro de estos modelos para las cuatro ecuaciones. Desde esta perspectiva, el nivel de estudios del trabajador influye positivamente en la calidad del empleo, al aumentar las posibilidades de obtener mejores puestos de trabajo a 
medida que aumenta su nivel de educación. Esta perspectiva es coherente con la literatura que aborda el nivel de educación como motor de perfeccionamiento del trabajo. Los mejores patrones de instrucción de la mano de obra se traducen en puestos de trabajo mejor remunerados, mayores posibilidades de formalización y mejores condiciones de trabajo. Así, las inversiones en educación y calificación del trabajo son bastante eficaces en términos de resultados y mejora de la calidad de las ocupaciones de los trabajadores, debido al comportamiento siempre sensible de la calidad del empleo en relación con la educación.

En el caso de la variable "calidad del empleo rezagada en un período", $I Q E_{t-1}$, el coeficiente es positivo y significativo a un nivel de significación de por lo menos el $5 \%$ en los cuatro modelos. Así, la calidad del empleo tiende a persistir de un año a otro. Se observa que la calidad del empleo pasada tiende a persistir más fuertemente en el empleo no agrícola urbano. Esto se debe a que, además de ser más avanzado que los demás, este mercado de trabajo presenta mayores niveles de formalización e incidencia de la legislación laboral. La lógica involucrada se basa en el comportamiento del trabajador y la propia dinámica del mercado de trabajo. En general, los trabajadores solo tienen incentivos para cambiar de puesto de trabajo si las condiciones ofrecidas en el empleo futuro son mejores que las del empleo actual, en un escenario de crecimiento del producto y reducción del desempleo, como el que caracterizó el período analizado, en el que la tasa media de desempleo abierto disminuyó del 11,48\% en 2004 al 4,8\% en 2014, conforme datos del IBGE. Así, el contexto macroeconómico del período tiene una gran influencia en los resultados presentados aquí 4 . Se argumenta también que el mercado de trabajo presenta cierta resistencia a la baja en términos de reducción de las conquistas laborales. En general, los derechos adquiridos no se pierden con el paso del tiempo. Este comportamiento también se refleja en la calidad del empleo, en la medida en que se crea cierta resistencia cuando se trata de condiciones mejores de trabajo ya adquiridas. Esto causa la persistencia de la calidad del empleo y su influencia en el empleo actual.

\section{Consideraciones finales}

En este estudio se buscó evaluar la evolución de la calidad del empleo para cuatro grupos de trabajadores, creando un paralelo como forma de evaluar las asimetrías existentes entre ellos. Se aplicó una metodología específica de verificación de los factores condicionantes de la calidad del empleo para los grupos seleccionados con el propósito de ampliar la base de explicación de las asimetrías verificadas.

Como es sabido, las recientes transformaciones del espacio, ya sea urbano o rural, tuvieron importantes efectos en las relaciones de trabajo. Sin embargo, en esta investigación se observa que esos cambios van mucho más allá de las nuevas dinámicas de producción del universo rural. En la actualidad, el comportamiento del empleo rural obedece a una pluralidad de condicionantes que van mucho más allá de los que tienen lugar en el espacio rural. Las dinámicas urbanas también adquieren importancia y comienzan a tenerse en cuenta para evaluar con mayor precisión la trayectoria de las relaciones de trabajo rurales.

El índice de calidad del empleo propuesto en esta investigación evidenció una profunda heterogeneidad en las relaciones de trabajo en los espacios estudiados. En general, los trabajadores agrícolas tienen condiciones de trabajo más precarias que sus pares no agrícolas. También con respecto a la asimetría en el mercado de trabajo, cabe señalar las diferencias existentes entre el empleo urbano y el rural. El universo rural persiste como un ambiente más precario en comparación con el medio urbano, aunque las diferencias estén disminuyendo con el tiempo.

\footnotetext{
4 Se recuerda que los resultados de la investigación no captan los efectos de la reciente crisis económica, social y política en el Brasil, que resultan más intensos a partir de 2015.
} 
Los espacios subnacionales también presentan algunas especificidades. En general, las mejores condiciones de trabajo y las dinámicas más intensas y avanzadas se encuentran en el complejo agroexportador de la región centro-sur del Brasil. Esto contrasta sobre todo con la situación en el Nordeste septentrional, que se encuentra aún desconectado de este sistema y presenta profundas diferencias con respecto al complejo agroindustrial del centro-sur. Son evidentes las relaciones de trabajo precarias que caracterizan a la región.

Por último, del análisis de los modelos presentados surge que, en general, el crecimiento económico se refleja en mayor medida en el empleo agrícola. La dinámica de las actividades agrícolas influye positivamente en el nivel de calidad del empleo agrícola. Sin embargo, el crecimiento de la producción agrícola no parece afectar de modo significativo la calidad del empleo no agrícola, incluso en un contexto de intensificación de las agroindustrias en el Brasil. Por otra parte, el crecimiento de la producción no agrícola tiene, por regla general, un impacto negativo en la calidad del empleo en la agricultura, pues constituye una expansión de la frontera de posibilidades para los trabajadores rurales. Como se mostró en este trabajo, esto contribuye al envejecimiento de la población rural ocupada en las actividades agrícolas e incluso perjudica la viabilidad y la continuidad de las empresas agrícolas.

En el caso del empleo no agrícola se observa una dinámica diferente. El crecimiento económico reciente ha extendido las mismas formas de contratación ya verificadas, sin grandes cambios estructurales en los mercados de trabajo no agrícola, o, en algunos casos, ha generando empleos en la base con peores índices de calidad. Sin embargo, es necesario hacer una salvedad importante: el comportamiento del mercado de trabajo a largo plazo puede pasar a responder a una dinámica diferente de la verificada hasta ahora. Esto se debe a que, a medida que la economía se acerca al pleno empleo inducida por el crecimiento económico, la extensión de las mismas formas de contratación a los trabajadores desocupados se vuelve cada vez más difícil. Así, a medida que el crecimiento económico se desarrolla, incluso a largo plazo, se espera que provoque cambios en la estructura del mercado de trabajo. No obstante, la posibilidad de cambios estructurales inducidos por el pleno empleo se ha vuelto remota dada la reciente crisis en el Brasil.

La investigación también mostró que el aumento de los ingresos del trabajo y el nivel de educación medio de los trabajadores son instrumentos importantes, no solo para aumentar los niveles de calidad del empleo, sino también como estrategia para superar algunos problemas de los mercados estudiados, como la heterogeneidad verificada entre los grupos.

\section{Bibliografía}

Arellano, M. y S. Bond (1991), "Some tests of specification for panel data: Monte Carlo evidence and an application to employment equations", The Review of Economic Studies, vol. 58, № 2, abril.

Arellano, M. y O. Bover (1995), "Another look at the instrumental variable estimation of error-components models", Journal of Econometrics, vol. 68, № 1, julio.

Balsadi, O. V. (2007), "Qualidade do emprego na agricultura brasileira no período 2001-2004 e suas diferenciações por culturas", Revista de Economia e Sociologia Rural, vol. 45, № 2, abril-junio.

Balsadi, O. V. y J. F. G. da Silva (2008), "A polarização da qualidade do emprego na agricultura brasileira no período 1992-2004", Economia e Sociedade, vol. 17, № 3, diciembre.

Balsadi, O. V. y M. E. Delgrossi (2018), "Labor and employment in Brazilian Northeastern agriculture: a look at the 2004-2014 period", Revista de Economia e Sociologia Rural, vol. 56, № 1, enero-marzo.

Baltagi, B. H. (2009), Econometric Analysis of Panel Data, $4^{a}$ edición, Chichester, Wiley.

Blundell, R. y S. Bond (1998), "Initial conditions and moment restrictions in dynamic panel data models", Journal of Econometrics, vol. 87, № 1, noviembre.

Campolina, B., F. G. Silveira y L. C. de Magalhães (2009), "O mercado de trabalho rural: evolução recente, composição da renda e dimensão regional”, Texto para Discussão, № 1398, Río de Janeiro, Instituto de Investigación Económica Aplicada (IPEA). 
Carneiro, M. J. (1998), "Ruralidade: novas identidades em construção", Estudos Sociedade e Agricultura, vol. 6, No 2, octubre.

Da Silva, J. G. (1997), "O novo rural brasileiro", Nova Economia, vol. 7, № 1, mayo.

Do Nascimento, C. A. y otros (2008), "A qualidade do emprego rural na região nordeste (2002 e 2005)", Revista da $A B E T$, vol. 7, № 2, diciembre.

Dos Passos, M. M., R. Bariou y V. Dubreuil (2006), "Evolução da fronteira agrícola no centro-oeste do Mato Grosso", Geosul, vol. 21, № 41, enero-junio.

Fajardo, S. (2008), "Complexo agroindustrial, modernização da agricultura e participação das cooperativas agropecuárias no estado do Paraná", Caminhos de Geografia, vol. 9, № 27, septiembre.

Freguglia, R. da S., E. Gonçalves y E. R. da Silva, (2014), "Composition and determinants of the skilled out-migration in the Brazilian formal labor market: a panel data analysis from 1995 to 2006", Economia, vol. 15, № 1, marzo.

Kleibergen, F. y R. Paap (2006), "Generalized reduced rank tests using the singular value decomposition", Journal of Econometrics, vol. 133, № 1, julio.

Marinho, E. y J. Araujo (2010), "Pobreza e o sistema de seguridade social rural no Brasil", Revista Brasileira de Economia, vol. 64, № 2, abril-junio.

Priori, A. y otros (2012), História do Paraná: séculos XIX e XX, Maringá, Eduem. 